\title{
Experimental non-severe hypoglycaemia substantially impairs cognitive function in type 2 diabetes: a randomised crossover trial
}

\author{
Malin Nilsson ${ }^{1}$ (1) $\cdot$ Nicole Jensen ${ }^{1} \cdot$ Michael Gejl $^{2} \cdot$ Marianne L. Bergmann $^{3} \cdot$ Heidi Storgaard ${ }^{4} \cdot$ Mette Zander $^{1}$. \\ Kamilla Miskowiak ${ }^{5,6}$ • Jørgen Rungby ${ }^{1,2,7}$
}

Received: 13 March 2019 / Accepted: 21 June 2019 / Published online: 31 July 2019

(C) Springer-Verlag GmbH Germany, part of Springer Nature 2019

\begin{abstract}
Aims/hypothesis Previous studies have demonstrated a relationship between cognitive impairment and hypoglycaemia ( $<3 \mathrm{mmol} / \mathrm{l}$ ). This study hypothesised that non-severe insulin-induced hypoglycaemia reduces cognitive function in individuals with type 2 diabetes.

Methods In this randomised crossover study, 25 participants with type 2 diabetes attended two experimental visits with hyperinsulinaemic glucose clamping: one hypoglycaemic clamp (plasma glucose $3.0 \pm 0.2 \mathrm{mmol} / \mathrm{l}$ ) and one euglycaemic clamp (plasma glucose $6.0 \pm 0.2 \mathrm{mmol} / \mathrm{l}$ ). Participants were eligible if their diabetes was treated with diet or glucose-lowering medications (except sulfonylureas or insulin), age was 35-70 years, BMI was 23-35 kg/m² and HbA $1 \mathrm{c}$ was below $75 \mathrm{mmol} / \mathrm{mol}(9 \%)$. Cognitive function was assessed with a neurocognitive test battery measuring verbal memory, executive function, sustained attention and psychomotor speed. From the examined cognitive domains, a global cognition score was constructed estimating global cognition. A measurement for psychomotor speed was selected as the primary outcome. Participants and people assessing the outcomes were blinded to group assignment.

Results Cognitive performance was impaired during hypoglycaemia with a mean score in the primary outcome test, Symbol Digit Modalities Test measuring psychomotor speed, of $48.7 \pm 9.8$ (hypoglycaemia) vs $56.6 \pm 12.0$ (euglycaemia); i.e. a change of -7.9 points $(95 \% \mathrm{CI}-10.9,-4.9 ; p<0.0001)$. In addition, hypoglycaemia reduced global cognitive score by $-0.7(95 \% \mathrm{CI}$ $-0.9,-0.6 ; p<0.0001)$. A stable glucose plateau was achieved during both experimental visits. For the hypoglycaemic clamp, mean plasma glucose concentration $( \pm \mathrm{SD})$ during neurocognitive testing was $3.1( \pm 0.3) \mathrm{mmol} / \mathrm{l}$. Age, sex, fasting C-peptide, counter-regulatory hormones and the severity of hypoglycaemic symptoms did not influence cognitive function.

Conclusions/interpretation Acute non-severe hypoglycaemia (mean plasma glucose $3.1 \mathrm{mmol} / \mathrm{l}$ ) has a substantial negative impact on cognitive function in individuals with type 2 diabetes.

Trial registration ClinicalTrials.gov NCT03014011.

Funding The study was supported in part by a research grant from the Investigator Initiated Studies Program of Merck Sharp \& Dohme Corp (MSD-MA-NORD-007-01). The opinions expressed in this paper are those of the authors and do not necessarily represent those of Merck Sharp \& Dohme Corp. Funding was also received from Skibsreder Per Henriksen, R. og hustrus Foundation, The Danish Alzheimer Foundation and Savværksejer Jeppe Juhl og hustrus Foundation.
\end{abstract}

Electronic supplementary material The online version of this article (https://doi.org/10.1007/s00125-019-4964-4) contains peer-reviewed but unedited supplementary material, which is available to authorised users.

Malin Nilsson

malin.sofia.desiree.nilsson@regionh.dk

1 Department of Endocrinology, Bispebjerg University Hospital, Bispebjerg Bakke 23, 2400 Copenhagen, NV, Denmark

2 Department of Endocrinology, Aarhus University Hospital, Aarhus, Denmark

3 Department of Biochemistry and Immunology, University Hospital of Southern Denmark, Vejle, Denmark
Clinical Metabolic Physiology, Steno Diabetes Center Copenhagen, Gentofte Hospital, Hellerup, Denmark

5 Department of Psychology and Mental Health Services, University of Copenhagen, Copenhagen, Denmark

6 Psychiatric Centre Copenhagen, Rigshospitalet, Denmark

7 Copenhagen Center for Translational Research, Bispebjerg University Hospital, Copenhagen, Denmark 


\section{Research in context}

\section{What is already known about this subject?}

- Severe hypoglycaemia is known to impair cognitive function in healthy people and individuals with diabetes, thus affecting their day-to-day activity

- Hypoglycaemia is the major limiting factor for intensive glycaemic control in both type 1 and type 2 diabetes

What is the key question?

- Is cognitive function affected also by non-severe hypoglycaemia ( $\geq 3 \mathrm{mmol} / \mathrm{l})$ in type 2 diabetes?

\section{What are the new findings?}

- A hypoglycaemic episode (mean plasma glucose $3.1 \mathrm{mmol} / \mathrm{l}$ ) led to substantial impairment of all of the examined cognitive domains compared with euglycaemia $(5.8 \mathrm{mmol} / \mathrm{l})$, including psychomotor speed, attention, memory and executive function

- Reaction time was prolonged by approximately $20 \%$ during hypoglycaemia

- The effect of hypoglycaemia on global cognition was not influenced by age, sex, fasting C-peptide, counterregulatory hormones or the severity of hypoglycaemia-related symptoms

\section{How might this impact on clinical practice in the foreseeable future?}

- Based on our findings, non-severe hypoglycaemia should be considered a substantial risk factor for acute cognitive impairment in type 2 diabetes

Keywords Cognitive function $\cdot$ Hypoglycaemia $\cdot$ Type 2 diabetes

\author{
Abbreviations \\ ETD Estimated treatment difference \\ RAVLT Rey Auditory Verbal Learning Test \\ RVP Rapid Visual Processing \\ SDMT Symbol Digit Modalities Test \\ TMT Trail Making Test \\ WAIS-III Wechsler Adult Intelligence Scale III
}

\section{Introduction}

Hypoglycaemia is the major limiting factor for intensive glycaemic control in both type 1 and type 2 diabetes mellitus $[1,2]$. While hypoglycaemia differs in severity, it remains potentially harmful, impacting cognitive, metabolic, cardiac and neural processes [3]. Hypoglycaemia may also have a significant negative impact on quality of life $[4,5]$.

Cognitive function refers to all aspects of thinking and intellectual activity, and cognitive processes are fundamental for performing many everyday tasks. Even subtle changes in cognitive function can potentially influence a person's capacity to function optimally and cognitive impairment raises safety concerns for many occupational activities. In individuals with type 1 diabetes and healthy control groups, acute insulin-induced hypoglycaemia (i.e. plasma glucose levels of 2.2-3.1 mmol/l) has been widely studied and shown to impair performance across several cognitive tests (measuring working memory, attention and reaction time, among others) [6-10]. Acute cognitive decline during hypoglycaemia in individuals with type 1 diabetes can thus impair their ability to tackle cognitive challenges both in daily life and in occupational settings. Nevertheless, it is less well established whether individuals with type 2 diabetes will experience similar cognitive impairment from acute hypoglycaemia due to the underlying pathophysiological differences between type 1 and type 2 diabetes, such as the presence of insulin resistance, which may impact the brain negatively in type 2 diabetes [11]. Furthermore, methodological problems may limit comparisons between studies [12].

The effect of hypoglycaemia on cognitive function in type 2 diabetes above the level considered clinically important by the International Hypoglycaemia Study Group [3] is still unclear. The few reports on cognitive function at higher glucose levels $(\geq 3.0 \mathrm{mmol} / \mathrm{l})$ have focused on determining counterregulatory thresholds for symptomatic and hormonal responses of hypoglycaemia [13-16]. In these study designs, cognitive testing has been secondary and, although cognitive impairment has been demonstrated, the number of neurocognitive tests performed has been few, with no assessment of global cognitive function. Therefore, at glucose levels 
of $3.0 \mathrm{mmol} / \mathrm{l}$ and above, further clarification is needed as to whether cognitive function is affected in the type 2 diabetes population, and, if so, which cognitive domains are affected.

Here, we examined cognitive function during an acute, non-severe hypoglycaemic episode (target plasma glucose $3 \mathrm{mmol} / \mathrm{l}$ ) in participants with type 2 diabetes using an extensive neurocognitive test battery. By averaging scores from the examined cognitive domains, a global cognition score was constructed estimating global cognition. A measurement for psychomotor speed (Symbol Digit Modalities Test [SDMT]) was selected as the primary outcome, as it was previously proven to be sensitive to hypoglycaemia $[9,17]$. We hypothesised that non-severe insulin-induced hypoglycaemia would reduce processing speed (primary hypothesis) and global cognition (secondary hypothesis). Counter-regulatory hormones and C-peptide were determined for exploratory analyses.

\section{Methods}

Study population Individuals diagnosed with type 2 diabetes for at least 3 months were approached for participation. They were eligible if their diabetes was treated with diet or glucoselowering medications (except sulfonylureas or insulin), age was $35-70$ years, BMI was $23-35 \mathrm{~kg} / \mathrm{m}^{2}$ and $\mathrm{HbA}_{1 \mathrm{c}}$ was below $75 \mathrm{mmol} / \mathrm{mol}$ (9\%). Key exclusion criteria included recent ischaemic cardiac disease, severe diabetic complications or other major illnesses. Full inclusion and exclusion criteria are listed in the electronic supplementary material (ESM) Table 1. Randomisation was determined by sequential enrolment and lowest available number assignment. Participants were blinded to the sequence of glycaemic conditions and to their current blood glucose concentration.

All participants gave oral and written consent before participation. The study was designed and performed in accordance to the Helsinki Declaration of Good Clinical Practice and was approved by the local ethics committee. The study was registered at www.clinicaltrials.gov (ClinicalTrials.gov registration no. NCT03014011).

Study design Cognitive performance was measured during hypoglycaemic and euglycaemic conditions in a randomised, double-blinded, crossover study design (ESM Fig. 1). Hypoglycaemic (aiming for a plasma glucose target of $3.0 \pm$ $0.2 \mathrm{mmol} / \mathrm{l}$ ) and euglycaemic (plasma glucose clamp target $6.0 \pm 0.2 \mathrm{mmol} / \mathrm{l}$ ) clamps were performed on separate visits. The two experimental visits were separated by $21-42$ days to avoid effects of counter-regulatory hormone responses or other physiological effects of hypoglycaemia. Blood samples were drawn at each visit for determination of insulin, C-peptide, adrenaline (epinephrine), noradrenaline (norepinephrine), cortisol, growth hormone and glucagon; these were taken at arrival (baseline), 10 min prior to neurocognitive testing and after testing when glucose levels were normalised.

Experimental visit procedure Participants were instructed to maintain a regular diet and avoid alcohol and strenuous physical exercise 3 days prior to each experimental day to avoid extreme fluctuations in blood glucose levels. After an overnight fast, a cannula was inserted in an antecubital vein for blood sampling. The arm was kept heated with a heating pad throughout the examination to obtain vasodilation as a proxy for arterialisation. A second cannula was inserted into an antecubital vein in the contralateral arm for continuous infusion of glucose (20\%) and human soluble insulin (Actrapid, Novo Nordisk, Bagsværd, Denmark). After a 15 min baseline period, a 3 min priming regimen commenced. Then, insulin was infused at a constant rate of $100 \mathrm{mU} \mathrm{m}^{-2} \mathrm{~min}^{-1}$ and this continued throughout neurocognitive testing. A variable infusion of glucose was given simultaneously and adjusted to maintain target glucose levels. Blood sampling for glucose was made at $5 \mathrm{~min}$ intervals throughout the clamp procedure. Plasma glucose was measured by the glucose oxidase method, using a glucose analyser (Yellow Springs Instrument, Yellow Springs, OH, USA). Neurocognitive testing was initiated when glucose levels had been stable for $40 \mathrm{~min}$. When testing was completed, the participants were served a meal and glucose levels were monitored until euglycaemia was safely maintained (Fig. 1).

Neurocognitive test battery The test battery included the following neurocognitive tests: the Rey Auditory Verbal Learning Test (RAVLT) [18, 19] (verbal memory), the Trail Making Test (TMT) parts A and B [20] (psychomotor speed and set shifting/task switching [a measure of executive function]), the SDMT [21] (psychomotor speed), the Wechsler Adult Intelligence Scale III (WAIS-III) Letter-Number Sequencing test [22] (working memory), the verbal fluency test (letters S and D [23]; one aspect of executive function) and the Rapid Visual Processing (RVP) test from the Cambridge Neuropsychological Test Automated Battery (CANTAB) (sustained attention). To minimise learning effects between the two visits, alternate versions of the tests that are associated with greatest learning effects were used. The total duration of neurocognitive testing in each examination was approximately $45 \mathrm{~min}$.

During data analysis, four cognitive domains were constructed based on the neurocognitive tests. Global cognition was defined by the averaging of these domains into a global cognitive score [24].

As the primary outcome test assessing psychomotor speed we chose the SDMT (WPS; www.wpspublish.com). This test consists of a key of nine geometric elements, each associated with a number from 1 to 9 . The participant's score is the number of correct substitutions made within an interval of $120 \mathrm{~s}$. 


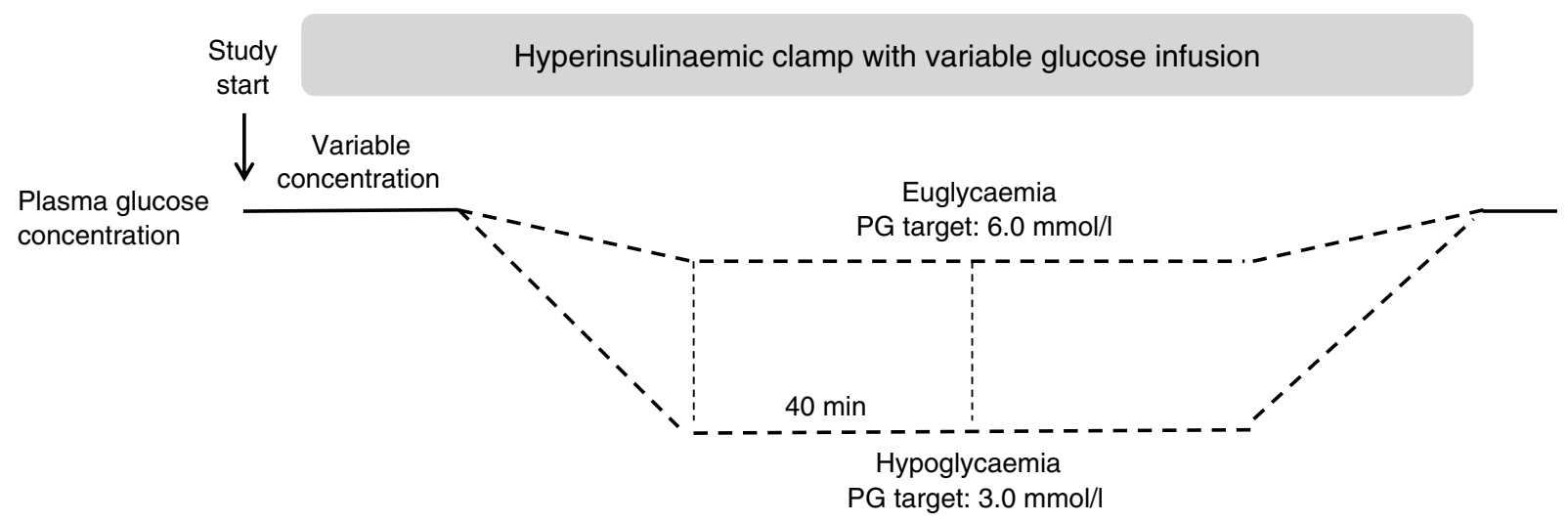

Neurocognitive
testing

Blood sampling

Fig. 1 Experimental visit design. Blood sampling times for noradrenaline, adrenaline, insulin, C-peptide, glucagon, cortisol and growth hormone are shown; blood was also sampled every $5 \mathrm{~min}$ for plasma glucose. PG, plasma glucose

Neurocognitive testing was conducted by research assistants from the Neurocognition and Emotion in Affective Disorders (NEAD) Group, Psychiatric Centre Copenhagen (www.neadgroup.org), who were blinded to the participants' glucose concentrations.

Hormones Counter-regulatory hormones were measured to ensure that the plasma glucose target was sufficiently low to elicit a counter-regulatory response. Plasma noradrenaline and adrenaline were analysed by LC-MS/MS. Plasma samples were extracted by solid phase extraction and the eluate was evaporated and reconstituted before analysis by LC-MS/MS. The analyses were calibrated by in-house prepared calibrators and the relative $\mathrm{SD}$ was below $10 \%$. Glucagon was measured by a commercially available ELISA kit (Mercodia, Uppsala, Sweden) according to the manufacturer's procedure. Three controls at various levels (Mercodia) were included in each assay. The relative SD of the assay controls was below $7 \%$ and in the range of the stated values. Insulin, C-peptide, growth hormone and cortisol were determined using the IMMULITE 2000 immunoassay system (Siemens Healthcare, Erlangen, Germany). All samples for a particular hormone were assayed in the same batch.

Symptoms of hypoglycaemia On each experimental visit, participants were informed of the characteristic symptoms of hypoglycaemia (as stated in the Edinburgh Hypoglycaemia Scale [25]) and instructed to inform the investigator of any symptom occurring during the clamp procedure.

Statistical analyses Based on the above neurocognitive test battery, four cognitive domains were constructed: verbal memory (consisting of RAVLT total recall across five trials, immediate recall following interference, delayed recall and recognition), executive function and working memory (consisting of TMT-B, WAIS-III letter-number sequencing, verbal fluency), sustained attention (consisting of RVP A', RVP mean latency) and psychomotor speed (consisting of SDMT and TMT-A). The domains were constructed by averaging standardised test scores for individual tests included in the domain. Standardisation was achieved using residuals from separate linear mixed-effect models with visit as fixed effect and participant as random effect, thereby minimising the influence of potential minor learning effects present during the second experimental visit. A global cognitive score was constructed by averaging the four domain scores [24].

For each glycaemic condition, the neurocognitive test scores were compared using linear mixed-effect models with glycaemic condition and visit interaction as fixed effects and participant as a random effect. Mean differences in cognitive function between hypoglycaemia and euglycaemia were estimated from the models and corresponding $95 \% \mathrm{CI}$ and $p$ values were calculated. Scores where larger values represent worse performance were inversed in the analyses of cognitive domains and global cognition. Bonferroni correction for multiple testing was applied, providing a level of significance of $p=0.0031$.

Effect sizes from individual tests were estimated from Cohen's $d$, calculated by the difference between the two glycaemic conditions divided by the residual SD.

Univariable linear regression analyses were performed to evaluate associations between changes in cognitive function (primary outcome, cognitive domains and global cognition) and the following variables: age, $\mathrm{HbA}_{1 \mathrm{c}}$, counter-regulatory hormones and fasting $\mathrm{C}$-peptide. The difference between sexes and hypoglycaemic symptom severity rating (none/mild vs moderate/severe) on change in cognitive function was analysed by two-sampled $t$ test. Further, logistic regression analyses were used to identify potential associations between hypoglycaemic symptom severity and the variables $\mathrm{HbA}_{1 \mathrm{c}}$, 
adrenaline, noradrenaline, growth hormone and cortisol. Model assumptions were validated by visual inspection of residuals and normal probability plots.

The primary endpoint of the study was the SDMT. In a previous trial [26], the SD for Digit Symbol Substitution Test (DSST, similar to SDMT) between hypoglycaemia and euglycaemia was 9 . We assumed a similar level of variance and opted to detect differences of 6 or more. A two-sided paired $t$ test and 5\% significance level suggested a sample size of 25 participants completing both experimental visits to achieve a $90 \%$ power to detect a true difference in cognitive function. A total of 28 participants were randomised to ensure that at least 25 participants completed the study. Graphical illustrations were performed using GraphPad Prism (version 7.02; GraphPad software; https://www.graphpad.com/ scientific-software/prism/) and statistical analyses were performed by R (version 3.2.3; http://cran.r-project.org/).

\section{Results}

Participants We screened 37 participants between May 2017 and July 2018, of whom 28 met inclusion criteria and were randomised, and 25 completed both experimental visits (ESM Fig. 2). Age was a mean \pm SD of $60 \pm 7.3$ years, BMI $30.5 \pm$ $3.4 \mathrm{~kg} / \mathrm{m}^{2}$ and $\mathrm{HbA}_{1 \mathrm{c}} 53.6 \pm 9.4 \mathrm{mmol} / \mathrm{mol}(7.1 \pm 0.9 \%)$. The participants' diabetes duration at inclusion was a mean \pm SD of $6.2 \pm 3.7$ years; data are presented for completers only (Table 1). Three participants (one man, two women) withdrew from the study after randomisation for reasons not related to

Table 1 Baseline characteristics of completers

\begin{tabular}{ll}
\hline Characteristic & Value \\
\hline Number of participants & 25 \\
Age, years & $60.2 \pm 7.3$ \\
BMI, $\mathrm{kg} / \mathrm{m}^{2}$ & $30.5 \pm 3.4$ \\
Race of European descent, $n(\%)$ & $25(100)$ \\
Sex, $n(\%)$ & \\
$\quad$ Female & $10(40)$ \\
$\quad$ Male & $15(60)$ \\
HbA ${ }_{1 c}$, mmol/mol & $53.6 \pm 9.4(41-70)$ \\
HbA ${ }_{1 \mathrm{c}}, \%$ & $7.1 \pm 0.9(5.9-8.6)$ \\
Duration of diabetes, years & $6.2 \pm 3.7(1-13.5)$ \\
Number of diabetic medications & $1.6(1-4)$ \\
Number of medications & $5.1 \pm 2.4(1-9)$ \\
Educational level, years & $13.6 \pm 2.9(9.5-19)$ \\
Systolic BP, mmHg & $133 \pm 12$ \\
Diastolic BP, mmHg & $79 \pm 7$ \\
Heart rate, beats/min & $70 \pm 14$ \\
\hline
\end{tabular}

Data are means \pm SD (minimum-maximum)

Baseline information collected at screening visit the intervention (difficulties with venous catheter [one], difficulties with fasting after completion of the first examination day [one] and withdrawal of consent prior to the first day of examination [one]). A stable glucose plateau was achieved during each condition and the individual glucose profiles are displayed in Fig. 2. Mean plasma glucose concentration ( \pm SD) during the neurocognitive testing was $3.13( \pm 0.27)$ $\mathrm{mmol} / \mathrm{l}$ for the hypoglycaemic clamp and $5.83( \pm 0.31)$ $\mathrm{mmol} / \mathrm{l}$ for the euglycaemic clamp.

Cognitive function For the primary outcome (SDMT), we found a statistically significant difference in performance with mean \pm SD SDMT scores of $48.7 \pm 9.8$ (hypoglycaemia) and $56.6 \pm 12.0$ (euglycaemia). The estimated treatment difference (ETD) was -7.9 (95\% CI $-10.9,-4.9 ; p<0.0001$; Cohen's $d=1.01$ ) (Table 2, Fig. 3a, b). Furthermore, global cognitive score showed a highly significant deterioration during the hypoglycaemic condition of -0.7 (95\% CI $-0.9,-0.6$; $p<0.0001$ ) (Table 2, Fig. 3c, d). Finally, analysis of the individual cognitive domains revealed that they were all substantially affected by hypoglycaemia, with varying effect sizes (verbal memory ETD -0.8 [95\% CI -1.1, -0.4], $p<0.0001$; executive function ETD -0.7 [95\% CI $-1.0,-0.4$ ], $p<0.0001$; psychomotor speed ETD -0.8 [95\% CI -1.1 , -0.5 ],$p<0.0001$; and sustained attention ETD -0.7 [95\% CI $-1.1,-0.3], p=0.0003$ ). The most substantial effect sizes (Cohen's $d>0.8$ ) were within the primary outcome, letternumber sequencing and mean latency, with performances deteriorating by $14 \%, 13 \%$ and $20 \%$, respectively.

Regression analyses revealed that poorer glycaemic control, indicated by increased $\mathrm{HbA}_{1 \mathrm{c}}$, was negatively associated with change in global cognitive score during hypoglycaemia ( $\beta-0.02 ; 95 \%$ CI $-0.04,-0.00 ; p=0.042$ ). Glycaemic control did not associate with other examined cognitive outcomes (i.e. primary outcome and cognitive domains) $(p>0.05)$. Further, no association was found between age, fasting Cpeptide or counter-regulatory hormones and change in cognitive function $(p>0.05)$, and no difference in change of cognitive function was found between sexes $(p>0.05)$.

Participants with moderate/severe symptom severity had a greater decrease in verbal memory during hypoglycaemia compared with participants with symptom severity rated as none/mild (mean difference of $-1.00 ; 95 \%$ CI $-1.64,-0.37$; $p=0.003$ ). No other cognitive domain, the primary outcome or global cognitive score differed between the two symptom severity ratings $(p>0.05)$. In addition, we examined a possible association between baseline $\mathrm{HbA}_{1 \mathrm{c}}$ and hypoglycaemic symptomatic severity, showing that odds of moderate/severe symptom severity during hypoglycaemia were increased by $13 \%$ (OR $1.13 ; 95 \%$ CI 1.02, 1.29; $p=0.035$ ) for each increase in $\mathrm{HbA}_{1 \mathrm{c}}$. Similar results were observed for adrenaline and noradrenaline, with the odds of moderate/severe hypoglycaemic symptom severity increasing with adrenaline 


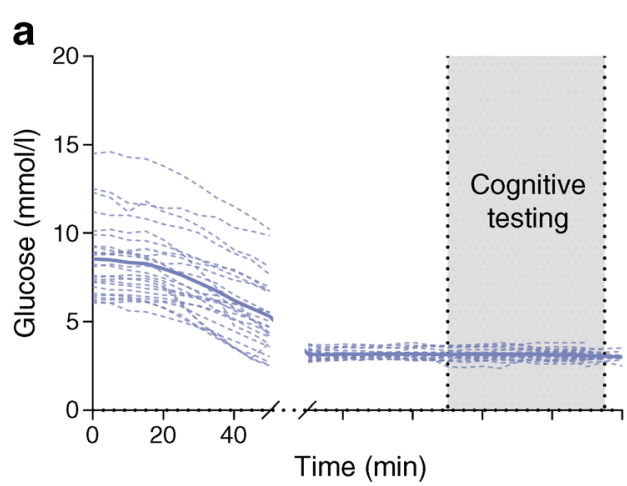

Fig. 2 Plasma glucose concentrations during (a) hypoglycaemia and (b) euglycaemia. The figures show glucose profiles during the first $50 \mathrm{~min}$ of the clamp procedure, during the $40 \mathrm{~min}$ period of stable glucose concentrations before cognitive testing and during cognitive testing. The $x$-axis

(OR 1.11; 95\% CI 1.04, 1.25; $p=0.021$ ) and noradrenaline (OR 9.50; 95\% CI 1.60, 147.31; $p=0.043$ ) responses. No association with symptom severity was found for cortisol or growth hormone $(p>0.05)$.

Hormonal responses Cortisol and growth hormone levels were increased during hypoglycaemia compared with

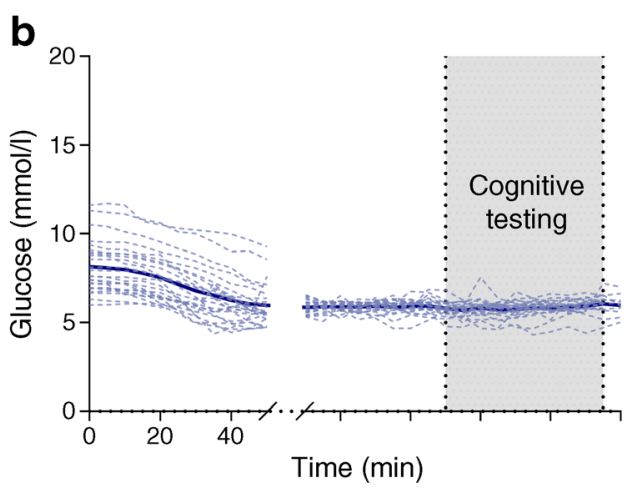

break represents time to establish stable glucose levels, which varied between participants. The solid blue line represents mean glucose concentration; dashed lines represent the individual glucose profiles

euglycaemia and this increase remained when euglycaemia was established after neurocognitive testing in the hypoglycaemic condition. The plasma levels of noradrenaline, adrenaline and glucagon also increased during hypoglycaemia compared with euglycaemia. C-peptide response was diminished during hypoglycaemia. There was no change in insulin levels between the two glycaemic conditions (Fig. 4).
Table 2 Cognitive assessment during hypoglycaemia and euglycaemia

\begin{tabular}{|c|c|c|c|c|c|c|}
\hline \multirow{2}{*}{$\begin{array}{l}\text { Neurocognitive tests and } \\
\text { cognitive domains }\end{array}$} & \multirow{2}{*}{$\begin{array}{l}\text { Euglycaemia } \\
\text { Mean ( } \pm \mathrm{SD})\end{array}$} & \multirow{2}{*}{$\begin{array}{l}\text { Hypoglycaemia } \\
\text { Mean }( \pm \mathrm{SD})\end{array}$} & \multicolumn{3}{|l|}{ ETD } & \multirow[t]{2}{*}{ ES } \\
\hline & & & Mean & $95 \% \mathrm{CI}$ & $p$ & \\
\hline Verbal memory & & & -0.8 & $-1.1,-0.4$ & $<0.0001$ & \\
\hline Total recall I-V & $47.7( \pm 7.4)$ & $43.1( \pm 10.9)$ & -4.7 & $-8.5,-1.0$ & 0.014 & 0.61 \\
\hline $\begin{array}{l}\text { Recall following } \\
\text { interference }\end{array}$ & $9.8( \pm 2.9)$ & $8.0( \pm 3.1)$ & -1.8 & $-2.6,-1.0$ & $<0.0001$ & 0.41 \\
\hline Delayed recall & $9.6( \pm 3.1)$ & $8.2( \pm 3.3)$ & -1.4 & $-2.4,-0.4$ & 0.005 & 0.69 \\
\hline Recognition & $13.8( \pm 1.4)$ & $12.0( \pm 2.8)$ & -1.9 & $-2.9,-0.8$ & 0.0006 & 0.78 \\
\hline Executive function & & & -0.7 & $-1.0,-0.4$ & $<0.0001$ & \\
\hline TMT-B, $\mathrm{s}^{\mathrm{a}}$ & $80.6( \pm 25.5)$ & $88.4( \pm 27.0)$ & 8.1 & $0.4,15.7$ & 0.040 & 0.53 \\
\hline $\begin{array}{l}\text { WAIS-III, letter-number } \\
\text { sequencing }\end{array}$ & $10.0( \pm 1.4)$ & $8.7( \pm 1.9)$ & -1.3 & $-2.1,-0.6$ & 0.0005 & 0.81 \\
\hline $\begin{array}{l}\text { Verbal fluency } \\
\text { (letters S+D) }\end{array}$ & $20.1( \pm 7.7)$ & $17.2( \pm 7.3)$ & -3.0 & $-4.9,-1.1$ & 0.002 & 0.73 \\
\hline Psychomotor speed & & & -0.8 & $-1.1,-0.5$ & $<0.0001$ & \\
\hline SDMT (primary outcome) & $56.6( \pm 12.0)$ & $48.7( \pm 9.8)$ & -7.9 & $-10.9,-4.9$ & $<0.0001$ & 1.01 \\
\hline TMT-A, s & $33.5( \pm 10.8)$ & $38.3( \pm 14.2)$ & 5.1 & $0.8,9.5$ & 0.020 & 0.57 \\
\hline Sustained attention ${ }^{\mathrm{b}}$ & & & -0.7 & $-1.1,-0.3$ & 0.0003 & \\
\hline RVP A' & $0.87( \pm 0.06)$ & $0.85( \pm 0.06)$ & -0.02 & $-0.05,0$ & 0.053 & 0.46 \\
\hline Mean latency, $\mathrm{ms}^{\mathrm{a}}$ & $438.3( \pm 85.1)$ & $526.1( \pm 127.5)$ & 90.2 & $42,138.4$ & 0.0002 & 0.83 \\
\hline Global cognitive score ${ }^{b}$ & & & -0.7 & $-0.9,-0.6$ & $<0.0001$ & \\
\hline
\end{tabular}

Mean scores from the neurocognitive tests and the four cognitive domains

Across all tests (except TMT-B, TMT-A and mean latency, where ETD values reflect time), negative ETD values reflect impaired performance during hypoglycaemia. After Bonferroni correction, the following neurocognitive tests were non-significant: RAVLT total recall and delayed recall, TMT-B, TMT-A and RVP A'

${ }^{\text {a }}$ Higher raw test score reflects worse performance

${ }^{\mathrm{b}}$ One missing data point due to technical difficulties; data analysed based on 49 tests results ES, estimated effect sizes by Cohen's $d$ 
Fig. 3 Results of (a, b) SDMT and $(\mathbf{c}, \mathbf{d})$ global cognitive score during hypoglycaemic and euglycaemic conditions. (a, b) Red represents participants where the number of correct answers during SDMT was lower during hypoglycaemia than euglycaemia. Blue represents participants where the number of correct answers during SDMT was lower during euglycaemia than hypoglycaemia. (c, d) Red represents participants where the global cognitive score was lower during hypoglycaemia than euglycaemia. Data from one participant are missing owing to technical difficulties

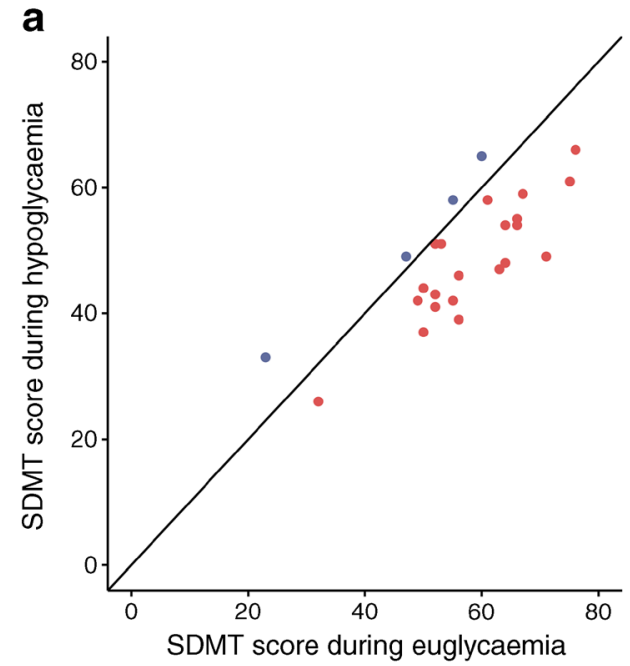

b

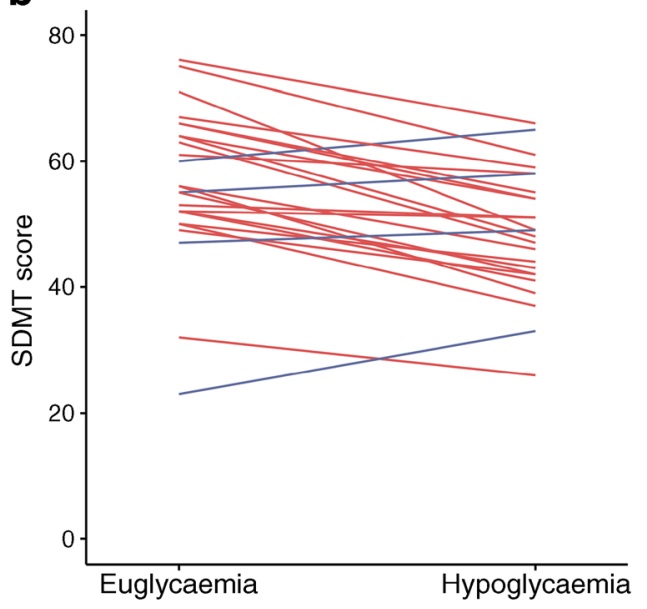

d

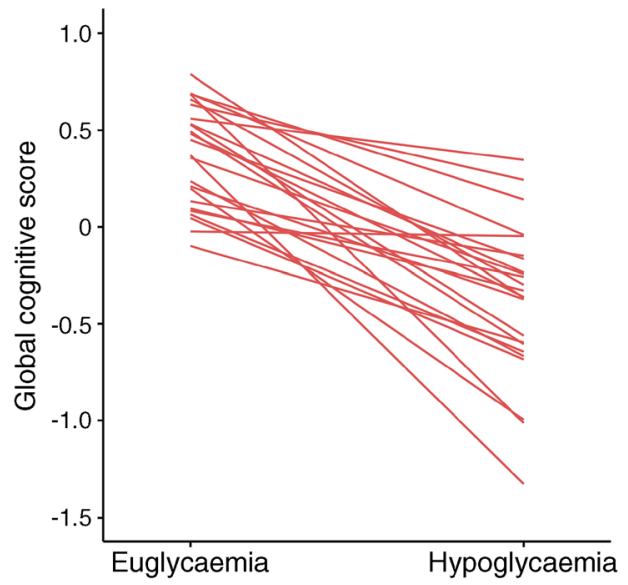

Symptoms and side effects Twenty of the 25 participants (80\%) reported symptoms related to hypoglycaemia (ESM Table 2). No examination was discontinued due to the reported hypoglycaemic symptoms. Based on symptoms reported by the participants and an assessment by the investigator, the severity of hypoglycaemic symptoms was categorised as 'none', 'mild', 'moderate' or 'severe'. One participant reported headache on the euglycaemic control day. Otherwise, there were no reported side effects on the control days.

\section{Discussion}

In this study, we demonstrate a substantial cognitive deterioration in participants with type 2 diabetes during hypoglycaemia compared with euglycaemia, with mean plasma glucose values of $3.1 \mathrm{mmol} / \mathrm{l}$ and $5.8 \mathrm{mmol} / \mathrm{l}$, respectively. The observed deterioration was highly significant for psychomotor speed (primary outcome) as well as global cognition (secondary outcome), and across all examined cognitive domains (verbal memory, executive function, sustained attention). Notably, a glucose level of $3.1 \mathrm{mmol} / \mathrm{l}$ is higher than the majority of previous studies assessing cognitive function during hypoglycaemia and slightly above the glycaemic threshold for impairment of cognitive function stated by the International Hypoglycaemia Study Group in 2017 [3]. Still, we demonstrate a significant and uniform impact of non-severe hypoglycaemia on cognitive function, and also after Bonferroni correction for multiple testing. This impact on cognitive function does not associate with age, fasting C-peptide or counter-regulatory hormones nor does it differ between sexes. This suggests that even non-severe hypoglycaemia in type 2 diabetes may impact on an individual's daily life, for instance by causing a deterioration in executive functions and a reduced ability to plan, organise and make decisions.

The estimated effect sizes (Cohen's $d$ ) for the cognitive domains demonstrated in this study are all equal to or above 0.7 , suggesting a moderate to high effect size. It is known that diabetes can lead to cognitive impairment: meta-analyses demonstrate cognitive deterioration in individuals with type 2 
Fig. 4 Counter-regulatory hormones during hypoglycaemia (black circles) and euglycaemia (white circles) at baseline, clamp (stable hypoglycaemia or euglycaemia) and recovery (after neurocognitive testing and at normalised blood glucose levels). (a) Insulin, (b) C-peptide, (c) cortisol, (d) growth hormone, (e) adrenaline, (f) noradrenaline and (g) glucagon. Data are presented as mean \pm SEM. $* p<0.05$ and $* * * p<0.001$ for hypoglycaemia vs euglycaemia
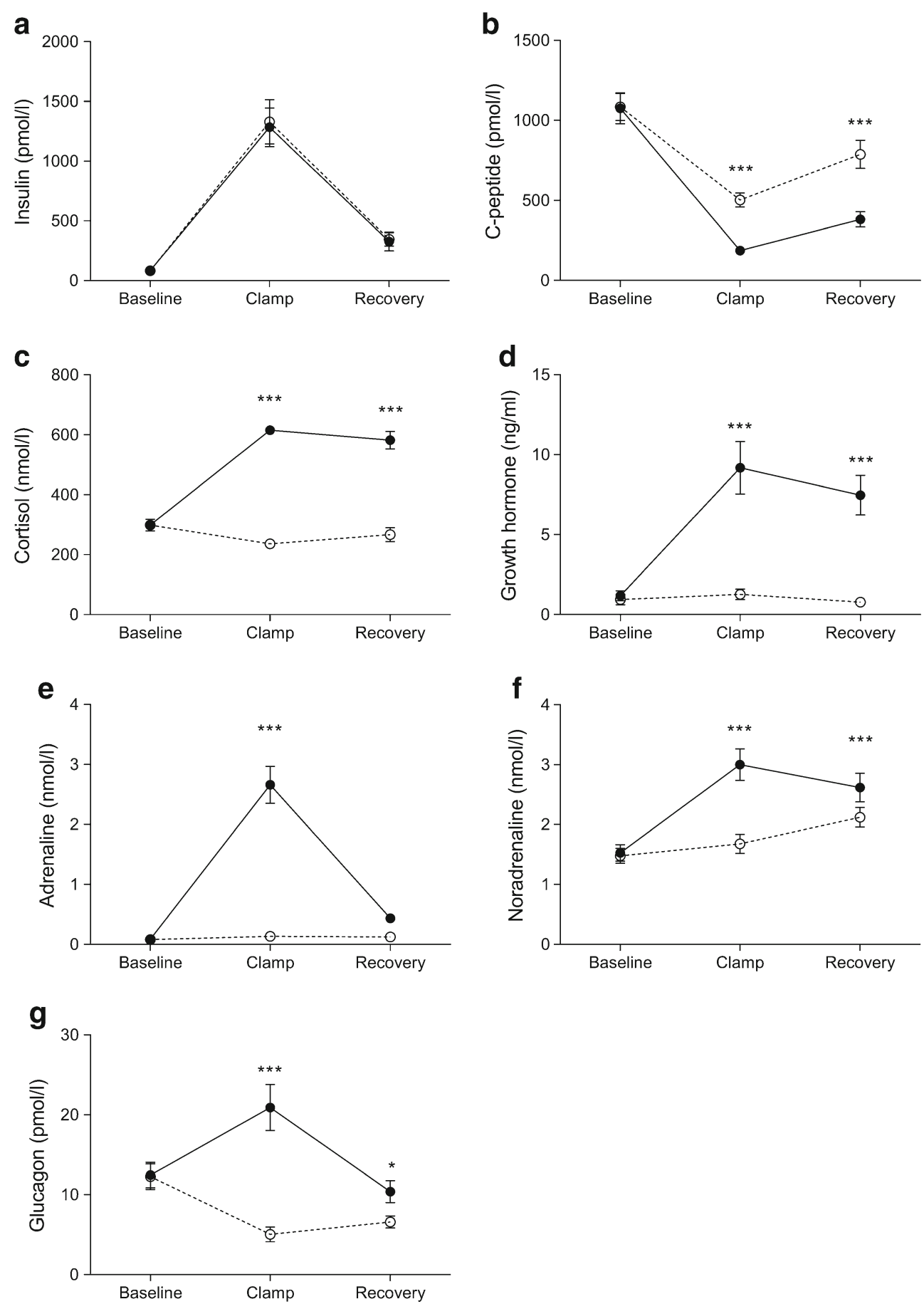

diabetes compared with healthy controls, with effects sizes ranging from -0.26 to -0.36 in several cognitive domains (motor function executive function, processing speed, verbal memory and visual memory) [27]. There is no consensus regarding a clinically relevant treatment effect size, but a change of 0.2-0.4 (small/moderate effect size) has been suggested [24]. We see the most substantial effect sizes within the domains of executive function, psychomotor speed and sustained attention, with the latter demonstrating an approximately $20 \%$ deterioration in reaction time. This delay in reaction time might be an important factor in many occupational activities, including the ability to drive. Impairment within the psychomotor speed domain has been identified as a predictor for driving assessment outcome, with lower SDMT scores seen in individuals that failed an on-road assessment [28].

Since insulin may also affect cognitive function [29, 30], we aimed to obtain similar plasma levels of insulin during the two conditions of neurocognitive testing. This goal was 
achieved. As expected, there was a significant difference in Cpeptide levels, indicating that endogenous insulin levels were suppressed during hypoglycaemia. The counter-regulatory hormone levels confirmed that a glucose level of $3.1 \mathrm{mmol} / 1$ is sufficient to induce a significant stress response, with increases in growth hormone, cortisol, noradrenaline and adrenaline.

This study shows that non-severe hypoglycaemia affects a broad range of cognitive functions: both simple functions, such as reaction time, and more complex functions such as memory and executive function. However, our design does not allow for a definition of glycaemic threshold for individual cognitive tests or overall cognitive impairment in type 2 diabetes, as it includes a single-step hypoglycaemic plateau only [31]. Our focus was to clearly establish whether cognitive impairment was present at a specific non-severe hypoglycaemic glucose level and, if so, which domains were affected. In addition, the population examined does not necessarily represent individuals that are at risk of hypoglycaemia, i.e. fairly good glycaemic control and no glucose-lowering treatment that could cause hypoglycaemia. Indeed, we chose to exclude participants treated with sulfonylureas and insulin to avoid potential episodes of hypoglycaemia in the days prior to examination, but we expect that druginduced hypoglycaemia would cause similar cognitive impairments. Furthermore, blood samples were collected from an antecubital vein, with the arm kept heated to obtain vasodilation, as a proxy for arterialisation. Ideally, arterial blood concentrations should be measured, but arterial cannulation is associated with difficulties and risks. This site of collection was chosen to ensure free movement of the hands for neurocognitive testing. Based on previous findings [32], we expect that our glucose values correspond well with glucose values obtained from arterialised venous blood from the hand. If blood glucose levels were underestimated by this procedure, the study would even further support our findings on cognitive function during hypoglycaemia. Finally, we did not test cognitive function during the recovery phase from hypoglycaemia, which has been examined in similar studies [9, 33, 34]. Findings of delayed recovery are not universal, and different cognitive tests seem to give different recovery times [35]. Here, we find that the demonstrated cognitive dysfunction during hypoglycaemia was substantial, and thus a potential delayed recovery phase should be considered for an individual's daily life.

Currently, there is no consensus suggesting a single test for measurement of cognitive function. The neurocognitive tests used in this study are well validated to detect cognitive dysfunction and examine the different cognitive domains that are particularly affected in diabetes $[27,36]$. Previous studies on cognitive function and acute hypoglycaemia have generally employed fewer tests for cognitive processes. Here, we chose a test battery of seven different neurocognitive tests for a comprehensive assessment of cognitive function and focused on multiple cognitive domains associated with diabetes. The
SDMT was selected as the primary outcome as it was previously proven to be sensitive to hypoglycaemia $[9,17]$. This was corroborated by our results as the SDMT had the highest estimated effect size, with a value of 1.01 .

Previous studies of hypoglycaemia have focused primarily on individuals with type 1 diabetes and healthy control groups. Here, we focus on type 2 diabetes as there might be differences in cognitive function between these groups. Hypoglycaemia is more frequent in asymptomatic individuals with type 2 diabetes than generally anticipated [2], making it important to clarify the effects of hypoglycaemia in this population. In this study, we demonstrate that a non-severe hypoglycaemic episode acutely impairs cognitive function. The long-term effects of non-severe glycaemic episodes have yet to be determined; however, evidence supports that severe hypoglycaemic episodes increase the risk of dementia and may cause permanent neural damage [37, 38]. Both type 1 and type 2 diabetes are associated with a greater risk of mild cognitive impairment and dementia [39], but the specific mechanism underlying the relationship between glycaemic control and the complications and comorbidities of diabetes and an increased risk of dementia is unknown [40]. Since repeated hypoglycaemia itself may increase the risk of dementia [41] and since impaired cognitive function increases the risk of hypoglycaemic events [42, 43], the relationship between hypoglycaemia and dementia may be bidirectional.

Exploratory analyses showed that with poorer glycaemic control there was a greater difference in the global cognition between hypoglycaemia and euglycaemia. This contrasts with other studies in which glycaemic control did not influence the effect of hypoglycaemia $(2.2 \mathrm{mmol} / \mathrm{l})$ on cognitive function in individuals with type 1 diabetes and poor glycaemic control (mean $\mathrm{HbA}_{1 \mathrm{c}}$ of $86 \mathrm{mmol} / \mathrm{mol}$ [10\%]) [6]. Moreover, KorzonBurakowska et al observed no significant difference in the glucose threshold for cognitive dysfunction when improving glycaemic control with insulin [15]. In the present study, there was no association between $\mathrm{HbA}_{1 \mathrm{c}}$ and the individual cognitive domains. Furthermore, poorer glycaemic control and higher adrenaline and noradrenaline responses increased the odds for moderate/severe symptom severity during hypoglycaemia.

In all, we found that participants with higher $\mathrm{HbA}_{1 \mathrm{c}}$ were more likely to have moderate/severe symptoms of hypoglycaemia and a greater difference in their global cognition performance compared with euglycaemia. Further exploratory analyses did not support a direct association between hypoglycaemia symptom severity and global cognition, suggesting that the symptoms of hypoglycaemia did not affect global cognitive performance in this population.

Further analyses of hypoglycaemic symptom severity and cognitive domains showed an association with verbal memory, as measured by RAVLT, which is an unstructured listlearning task that examines cognitive ability for learning, encoding and retrieval. None of the other cognitive domains 
were associated with the severity of hypoglycaemia-related symptoms. These analyses must be treated with caution since our evaluation of hypoglycaemic symptoms was based on partly objective investigator reporting. Our results suggest that several cognitive domains are affected by low levels of glucose and that the severity of hypoglycaemia-related symptoms does not influence cognitive impairment. Thus, individuals may continue with their daily activities unaware of the negative effect on cognitive performance. We suggest an increased focus on the impact of non-severe hypoglycaemia on cognition, and the potentially harmful implications on the activities of daily life.

In conclusion, acute hypoglycaemia (mean plasma glucose $3.1 \mathrm{mmol} / \mathrm{l}$ ) negatively affects cognitive function in individuals with type 2 diabetes. Therefore, this degree of hypoglycaemia should be considered a substantial risk factor in the treatment of diabetes, and clinicians should attempt to minimise hypoglycaemia when aiming for a patient's target glycaemic control.

Acknowledgements The authors would like to thank all of the study participants. They also thank H. Wodschow (University of Copenhagen, Denmark) and the research assistants from the Neurocognition and Emotion in Affective Disorders (NEAD) Group, Psychiatric Centre Copenhagen, Denmark, for assistance during the experimental visits. Thank to I. Brandslund and D. Olsen (Department of Biochemistry and Immunology, University Hospital of Southern Denmark) for performing the ELISA for glucagon. Finally, thanks to B. Della Valle (GLX Analytix, Copenhagen, Denmark) for proofreading the manuscript.

Data availability The datasets generated and/or analysed during the current study are available from the corresponding author on reasonable request.

Funding The study was supported in part by a research grant from the Investigator Initiated Studies Program of Merck Sharp \& Dohme Corp (MSD-MA-NORD-007-01). The opinions expressed in this paper are those of the authors and do not necessarily represent those of Merck Sharp \& Dohme Corp. Funding was also received from Skibsreder Per Henriksen, R. og hustrus Foundation, The Danish Alzheimer Foundation and Savværksejer Jeppe Juhl og hustrus Foundation. The funding sources were not involved in the design of the study; the collection, analysis, and interpretation of data; writing the report; or the decision to submit the report for publication.

Duality of interest The authors declare that there is no duality of interest associated with this manuscript.

Contribution statement $\mathrm{MN}$ contributed to trial design, collected the data, performed the data analyses and wrote the manuscript. NJ contributed to the statistical analyses, and review and editing of the manuscript. $\mathrm{MG}, \mathrm{HS}$ and $\mathrm{MZ}$ contributed to the study design and interpretation of data and critically reviewed the manuscript. MLB was involved in data analysis and revision of the manuscript. KM adapted the neurocognitive test battery, trained and supervised the research assistants who performed the neurocognitive testing, contributed to the statistical analysis plan and interpretation of results, and revised the manuscript. JR (study sponsor) was responsible for the overall supervision and design of the study, interpretation of data, and review and editing of the manuscript, and made the decision to submit it for publication. All authors approved the final version. JR is responsible for the integrity of the work as a whole.

\section{References}

1. Cryer PE (2002) Hypoglycaemia: the limiting factor in the glycaemic management of type I and type II diabetes. Diabetologia 45(7):937-948. https://doi.org/10.1007/s00125-0020822-9

2. Gehlaut RR, Dogbey GY, Schwartz FL, Marling CR, Shubrook JH (2015) Hypoglycemia in type 2 diabetes - more common than you think: a continuous glucose monitoring study. J Diabetes Sci Technol 9(5):999-1005. https://doi.org/10.1177/ 1932296815581052

3. International Hypoglycaemia Study Group (2017) Glucose concentrations of less than $3.0 \mathrm{mmol} / \mathrm{L}(54 \mathrm{mg} / \mathrm{dL})$ should be reported in clinical trials: a joint position statement of the American Diabetes Association and the European Association for the Study of Diabetes. Diabetes Care 40(1):155-157. https://doi.org/10.2337/ dc16-2215

4. Wild D, von Maltzahn R, Brohan E, Christensen T, Clauson P, Gonder-Frederick L (2007) A critical review of the literature on fear of hypoglycemia in diabetes: implications for diabetes management and patient education. Patient Educ Couns 68(1):10-15. https://doi.org/10.1016/j.pec.2007.05.003

5. Davis RE, Morrissey M, Peters JR, Wittrup-Jensen K, KennedyMartin T, Currie CJ (2005) Impact of hypoglycaemia on quality of life and productivity in type 1 and type 2 diabetes. Curr Med Res Opin 21(9):1477-1483. https://doi.org/10.1185/ 030079905X61929

6. Draelos MT, Jacobson AM, Weinger K et al (1995) Cognitive function in patients with insulin-dependent diabetes mellitus during hyperglycemia and hypoglycemia. Am J Med 98(2):135-144. https:// doi.org/10.1016/S0002-9343(99)80397-0

7. Deary IJ, Sommerfield AJ, McAulay V, Frier BM (2003) Moderate hypoglycaemia obliterates working memory in humans with and without insulin treated diabetes. J Neurol Neurosurg Psychiatry 74(2):278-279. https://doi.org/10.1136/jnnp.74.2.278-a

8. McAulay V, Deary IJ, Sommerfield AJ, Frier BM (2006) Attentional functioning is impaired during acute hypoglycaemia in people with type 1 diabetes. Diabet Med 23(1):26-31. https:// doi.org/10.1111/j.1464-5491.2005.01795.x

9. Gejl M, Gjedde A, Brock B et al (2018) Effects of hypoglycaemia on working memory and regional cerebral blood flow in type 1 diabetes: a randomised, crossover trial. Diabetologia 61(3):551561. https://doi.org/10.1007/s00125-017-4502-1

10. Maran A, Crepaldi C, Trupiani S et al (2000) Brain function rescue effect of lactate following hypoglycaemia is not an adaptation process in both normal and type I diabetic subjects. Diabetologia 43(6): 733-741. https://doi.org/10.1007/s001250051371

11. Ma L, Wang J, Li Y (2015) Insulin resistance and cognitive dysfunction. Clin Chim Acta 444:18-23. https://doi.org/10.1016/j.cca. 2015.01.027

12. Warren RE, Frier BM (2005) Hypoglycaemia and cognitive function. Diabetes Obes Metab 7(5):493-503. https://doi.org/10.1111/j. 1463-1326.2004.00421.x

13. Choudhary P, Lonnen K, Emery CJ et al (2009) Comparing hormonal and symptomatic responses to experimental hypoglycaemia in insulin- and sulphonylurea-treated type 2 diabetes. Diabet Med 26(7):665-672. https://doi.org/10.1111/j.1464-5491.2009.02759.x

14. Bremer JP, Baron M, Peters H et al (2006) Hormonal, subjective, and neurocognitive responses to brief hypoglycemia in postmenopausal women and age-matched men with type 2 diabetes mellitus. Metabolism 55(3):331-338. https://doi.org/10.1016/j.metabol. 2005.09.006

15. Korzon-Burakowska A, Hopkins D, Matyka K et al (1998) Effects of glycemic control on protective responses against hypoglycemia 
in type 2 diabetes. Diabetes Care 21(2):283-290. https://doi.org/10. 2337/diacare.21.2.283

16. Spyer G, Hattersley AT, MacDonald IA, Amiel S, MacLeod KM (2000) Hypoglycaemic counter-regulation at normal blood glucose concentrations in patients with well controlled type-2 diabetes. Lancet 356(9246):1970-1974. https://doi.org/10.1016/S01406736(00)03322-5

17. Gold AE, Deary IJ, MacLeod KM, Thomson KJ, Frier BM (1995) Cognitive function during insulin-induced hypoglycemia in humans: short-term cerebral adaptation does not occur. Psychopharmacology 119(3):325-333. https://doi.org/10.1007/ BF02246299

18. Corwin J, Bylsma FW (1993) Psychological examination of traumatic encephalopathy. Clin Neuropsychol 7(1):3-21. https://doi. org/10.1080/13854049308401883

19. Rey A (1964) L'examen clinique en psychologie. Presses Universitaires de France, Paris

20. Army Individual Test Battery (1944) Manual of directions and scoring. War Department AGsO, Washington, DC

21. Smith A (1973) Symbol Digit Modalities Test. Western Psychological Services, Los Angeles

22. Wechsler D (1997) Wechsler Adult Intelligence Scale, 3rd edn. The Psychological Corporation, San Antonio

23. Borkowski JG, Benton AL, Spreen O (1967) Word fluency and brain damage. Neuropsychologia 5(2):135-140. https://doi.org/10. 1016/0028-3932(67)90015-2

24. Miskowiak KW, Burdick KE, Martinez-Aran A et al (2017) Methodological recommendations for cognition trials in bipolar disorder by the International Society for Bipolar Disorders Targeting Cognition Task Force. Bipolar Disord 19(8):614-626. https://doi.org/10.1111/bdi.12534

25. Deary IJ, Hepburn DA, MacLeod KM, Frier BM (1993) Partitioning the symptoms of hypoglycaemia using multi-sample confirmatory factor analysis. Diabetologia 36(8):771-777. https:// doi.org/10.1007/BF00401150

26. Koehler G, Heller S, Korsatko S et al (2014) Insulin degludec is not associated with a delayed or diminished response to hypoglycaemia compared with insulin glargine in type 1 diabetes: a double-blind randomised crossover study. Diabetologia 57(1):40-49. https://doi. org/10.1007/s00125-013-3056-0

27. Palta P, Schneider AL, Biessels GJ, Touradji P, Hill-Briggs F (2014) Magnitude of cognitive dysfunction in adults with type 2 diabetes: a meta-analysis of six cognitive domains and the most frequently reported neuropsychological tests within domains. J Int Neuropsychol Soc 20(3):278-291. https://doi.org/10.1017/ S1355617713001483

28. Worringham CJ, Wood JM, Kerr GK, Silburn PA (2006) Predictors of driving assessment outcome in Parkinson's disease. Mov Disord 21(2):230-235. https://doi.org/10.1002/mds.20709

29. Novak V, Milberg W, Hao Y et al (2014) Enhancement of vasoreactivity and cognition by intranasal insulin in type 2 diabetes. Diabetes Care 37(3):751-759. https://doi.org/10.2337/dc13-1672

30. Kern W, Peters A, Fruehwald-Schultes B, Deininger E, Born J, Fehm HL (2001) Improving influence of insulin on cognitive functions in humans. Neuroendocrinology 74(4):270-280. https://doi. $\operatorname{org} / 10.1159 / 000054694$
31. Mitrakou A, Ryan C, Veneman Tet al (1991) Hierarchy of glycemic thresholds for counterregulatory hormone secretion, symptoms, and cerebral dysfunction. Am J Phys 260:E67-E74

32. Liu D, Moberg E, Kollind M, Lins PE, Adamson U, Macdonald IA (1992) Arterial, arterialized venous, venous and capillary blood glucose measurements in normal man during hyperinsulinaemic euglycaemia and hypoglycaemia. Diabetologia 35(3):287-290. https://doi.org/10.1007/BF00400932

33. Evans ML, Pernet A, Lomas J, Jones J, Amiel SA (2000) Delay in onset of awareness of acute hypoglycemia and of restoration of cognitive performance during recovery. Diabetes Care 23(7):893897. https://doi.org/10.2337/diacare.23.7.893

34. Herold KC, Polonsky KS, Cohen RM, Levy J, Douglas F (1985) Variable deterioration in cortical function during insulin-induced hypoglycemia. Diabetes 34(7):677-685. https://doi.org/10.2337/ diab.34.7.677

35. Zammitt NN, Warren RE, Deary IJ, Frier BM (2008) Delayed recovery of cognitive function following hypoglycemia in adults with type 1 diabetes: effect of impaired awareness of hypoglycemia. Diabetes 57(3):732-736. https://doi.org/10.2337/db07-0695

36. Ryan CM, Freed MI, Rood JA, Cobitz AR, Waterhouse BR, Strachan MW (2006) Improving metabolic control leads to better working memory in adults with type 2 diabetes. Diabetes Care 29(2):345-351. https://doi.org/10.2337/diacare.29.02.06.dc051626

37. Bree AJ, Puente EC, Daphna-Iken D, Fisher SJ (2009) Diabetes increases brain damage caused by severe hypoglycemia. Am J Physiol Endocrinol Metab 297(1):E194-E201. https://doi.org/10. 1152/ajpendo.91041.2008

38. Whitmer RA, Karter AJ, Yaffe K, Quesenberry CP Jr, Selby JV (2009) Hypoglycemic episodes and risk of dementia in older patients with type 2 diabetes mellitus. JAMA 301(15):1565-1572. https://doi.org/10.1001/jama.2009.460

39. Cheng G, Huang C, Deng H, Wang H (2012) Diabetes as a risk factor for dementia and mild cognitive impairment: a meta-analysis of longitudinal studies. Intern Med J 42(5):484-491. https://doi.org/ 10.1111/j.1445-5994.2012.02758.x

40. Mayeda ER, Whitmer RA, Yaffe K (2015) Diabetes and cognition. Clin Geriatr Med 31(1):101-1ix. https://doi.org/10.1016/j.cger. 2014.08.021

41. Mehta HB, Mehta V, Goodwin JS (2017) Association of hypoglycemia with subsequent dementia in older patients with type 2 diabetes mellitus. J Gerontol A Biol Sci Med Sci 72:1110-1116

42. de Galan BE, Zoungas S, Chalmers J et al (2009) Cognitive function and risks of cardiovascular disease and hypoglycaemia in patients with type 2 diabetes: the Action in Diabetes and Vascular Disease: Preterax and Diamicron Modified Release Controlled Evaluation (ADVANCE) trial. Diabetologia 52(11):2328-2336. https://doi.org/10.1007/s00125-009-1484-7

43. Feinkohl I, Aung PP, Keller M et al (2014) Severe hypoglycemia and cognitive decline in older people with type 2 diabetes: the Edinburgh Type 2 Diabetes Study. Diabetes Care 37(2):507-515. https://doi.org/10.2337/dc13-1384

Publisher's note Springer Nature remains neutral with regard to jurisdictional claims in published maps and institutional affiliations. 